\title{
La pratique des méthodes de détection du radon par les plastiques détecteurs de traces nucléaires
}

\author{
J. ANDRU*
}

(Manuscrit reçu le 21 avril 1998, révisé le 7 juin 1998, accepté le 22 juin 1998)

RÉSUMÉ S'il n'y avait pas eu un gaz (le radon) au milieu de la famille radioactive de l'uranium, la radioactivité naturelle n'aurait posé aucun problème sanitaire. Plus rare, le gaz thoron, son isotope, est négligé par la législation, mais ses descendants peuvent parfois devenir dangereux, souvent à cause de certains matériaux de construction. Les « Détecteurs Solides de Traces Nucléaires » (DSTN) enregistrent les impacts individuels des particules alpha, sans perturber le milieu. Ces DSTN sont des plastiques, sélectionnés pour leur sensibilité aux particules alpha. Un simple traitement alcalin permet d'agrandir, de visualiser, donc de compter les traces obtenues. Placés dans de petits boîtiers passifs, ils deviennent des dosimètres bon marché, pour la mesure intégrée du radon et l'évaluation du risque sanitaire. Une examen pratique montre que tout n'est pas si simple. Le choix d'un dosimètre * ouvert » (DSTN à l'air libre) ou « fermé » (DSTN dans un boîtier) est discuté. Les différences pratiques sont expliquées par l'examen du passage progressif d'un dosimètre « ouvert » à un dosimètre « fermé ». Indépendamment de la facilité d'emploi, pour une meilleure estimation globale du risque naturel, mieux vaudrait mesurer « ouvert ", tandis que pour les mesures relatives aux sciences de la terre, mieux vaudrait mesurer « fermé ». Le débat, lui, est ouvert.

ABSTRACT Practical radon detection by Solid State Nuclear Track Detectors

If the radioactive uranium family had not included a gas (radon), natural radioactivity would pose no health problem. Its rarer isotope, thoron gas, is neglected by legislation, but its daughter products can sometimes become dangerous, often because of the use of certain building materials. Solid state track detectors (SSNT) record individual alpha particle impacts without influencing their surroundings. These SSNTs are plastics chosen for their sensitivity to alpha particles. A simple alkaline treatment makes it possible to amplify, display and therefore count the tracks obtained. Placed in small passive cases, they become cheap dosimeters for use in the integrated measurement of radon and evaluation of the health risk. A practical examination shows that things are not quite so simple. The choice of an open dosimeter (free air SSNT) or closed dosimeter (encased SSNT) is discussed. The practical differences are explained by studying the progressive change from an open dosimeter to a closed dosimeter. Irrespective of the ease of use, open measurement is better for a an overall assessment of the natural risk, while closed measurement is better for earth science measurements. The debate, for its part, is open. 


\section{Les recommandations}

Depuis février 1990, la C.E.E. (Journal officiel des Communautés européennes) recommande un équivalent de dose maximale de $20 \mathrm{mSv}$ annuel, pour le public, dans les bâtiments anciens. En pratique, elle traduit prudemment ce chiffre, à l'aide du « modèle d'exposition actuel », en une concentration moyenne annuelle de $400 \mathrm{~Bq} / \mathrm{m}^{3}$ de gaz radon, dans les bâtiments.

Plus récemment, l'ICRP 65 (1994) fixe la dose maximale à $10 \mathrm{mSv}$ par an, équivalente à une recommandation annuelle de 2,6 WLM (Working Level Month), soit, en unités SI, une exposition annuelle de $9,3 \mathrm{mJh} / \mathrm{m}^{3}$ aux descendants ambiants à vie courte. Cette recommandation en « exposition » correspond rigoureusement à la recommandation de $600 \mathrm{~Bq} / \mathrm{m}^{3}$ de gaz radon, à condition de supposer un facteur d'équilibre $F=0,4$ entre descendants et gaz, et un temps d'exposition annuelle de $7000 \mathrm{~h}$ (=80\% du temps).

Les « concentrations » sont en fait des activités volumiques $\mathrm{A}\left(\mathrm{Bq} / \mathrm{m}^{3}\right)$, c'est-àdire un nombre de particules alpha émis par seconde, dans $1 \mathrm{~m}^{3} \mathrm{~d}$ 'air. Ce n'est pas une quantité de radioélément. Plus la désintégration d'un radioélément est lente (donc sa vie longue), plus il en faut beaucoup pour atteindre une même activité. Par exemples : $-1000 \mathrm{~Bq}$ d'uranium correspond à $0,08 \mathrm{~g}$ d'uranium (L'uranium est très peu radioactif).

$-1000 \mathrm{~Bq}$ de radon correspond à 0,000000000000018 litre de gaz radon, soit pourtant 480 millions d'atomes. Même s'il avait une forte odeur, à $1000 \mathrm{~Bq} / \mathrm{m}^{3}$, le radon serait très loin d'être perceptible. Aucun polluant n'est traqué à $1,8 \times 10^{-11} \mathrm{ppm}$ !

$-1000 \mathrm{~Bq}$ de thoron correspond à encore 6000 fois moins que pour le radon! Soit infiniment peu de gaz, pas loin du zéro homéopathique bien connu !

À l'équilibre radioactif $(F=1)$, tous les radioéléments ont la même activité. Pour $F=0$, il n'y a que du gaz, donc pas d'énergie potentielle alpha (EPA en $\mu J$ oules) des descendants du radon, donc pas de risque sanitaire. Deux relations importantes définissent $F$, et relient les recommandations en énergie (Joules $/ \mathrm{m}^{3}$ ) à celles en concentration $\left(\mathrm{Bq} / \mathrm{m}^{3}\right)$ :

Pour ${ }^{222} \mathrm{Rn}$ (Radon) : $\quad \mathrm{EPA}\left(\mathrm{R}, \mu \mathrm{J} / \mathrm{m}^{3}\right)=5.5 \times 10^{-3} \cdot \mathrm{F}_{\mathrm{R}} \cdot \mathrm{A}\left(\mathrm{R}, \mathrm{Bq} / \mathrm{m}^{3}\right)$ Pour ${ }^{220} \mathrm{Rn}$ (Thoron) : $\mathrm{EPA}\left(\mathrm{T}, \mu \mathrm{J} / \mathrm{m}^{3}\right)=76.0 \times 10^{-3} \cdot \mathrm{F}_{\mathrm{T}} \cdot \mathrm{A}\left(\mathrm{T}, \mathrm{Bq} / \mathrm{m}^{3}\right)$

Notons, qu'à activité égale du gaz, l'EPA ${ }_{\mathrm{T}}$ thoron est supérieure à l'EPA $\mathrm{R}_{\mathrm{R}}$ radon. Mais, le risque thoron est compensé par un « $\mathrm{F}_{\mathrm{T}}$ 》 généralement très faible $\left(\mathrm{F}_{\mathrm{T}} \approx\right.$ 0,05 et $\mathrm{F}_{\mathrm{R}} \approx 0,40$ ) et par l'expectoration qui évacuerait 3 fois mieux certains descendants du thoron des bronches, avant leur désintégration, grâce à leur plus longue durée de vie $(\approx 10 \mathrm{~h}$ contre $30 \mathrm{~min})$.

Le gaz thoron est négligé par les recommandations. Bien que souvent justifié, cet oubli peut parfois aboutir à déclarer saine une maison qui ne l'est pas. Les excès de thoron proviennent plus souvent des émanations des murs que du sol (Steinhäuser, 1995; Li and Schery; Cliff et al., 1992). 
TABLEAU I

De la concentration de radon au risque sanitaire.

From radon concentration to health risk.

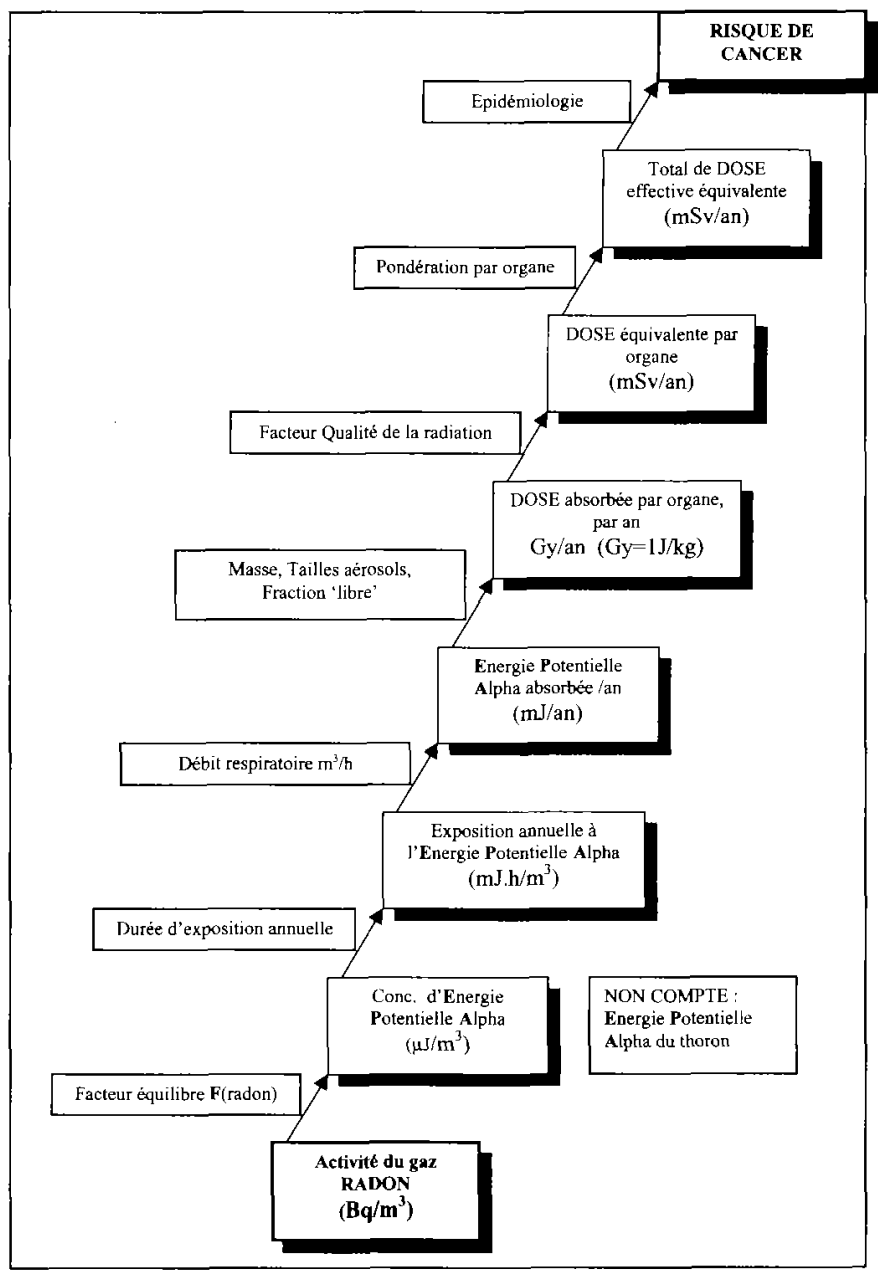

Le tableau I résume les principaux coefficients qui permettent de passer de la dose biologique en $\mathrm{mSv}$ aux diverses mesures pratiques. Entre autres, les facteurs durée d'exposition, débit respiratoire, $F$ et « fraction libre » apparaissent à différents niveaux.

Pour l'aspect sanitaire, l'objectif d'une première mesure (Brochure OMS, 1996) est d'évaluer globalement le risque. Mais comme, « la mesure du gaz radon 
ne permet pas directement d'accéder au risque radon » (Collectif IPSN, 1996), des recommandations en terme de descendants seraient préférables. Par contre, pour les sciences de la terre, une bonne métrologie du seul gaz radon est essentielle (géologie, hydrologie, volcanisme, études sismiques, etc.).

\section{Principes de fonctionnement des plastiques DSTN (détecteur solide de traces nucléaires)}

Dans l'air, chaque particule $\alpha$, perd rapidement son énergie initiale. À chaque émetteur $\alpha$ correspond donc une distance maximum, au-delà de laquelle elle disparaît, donc ne peut plus être enregistrée. Cette distance maximum d'enregistrement est de l'ordre de $35 \mathrm{~mm}$ pour le radon, et de l'ordre de $60 \mathrm{~mm}$ pour son descendant le plus énergétique.

Seuls les dosimètres bon marché nous intéressent ici, c'est-à-dire les dosimètres " passifs ». Ils fonctionnent sans circulation forcée de l'air. Il existe cependant quelques dosimètres « actifs » qui utilisent aussi des DTSN. Ils accumulent les poussières ambiantes sur des filtres, face à un DSTN qui enregistre les émissions alpha des descendants ainsi piégés.

Un DSTN, placé dans un boîtier passif, « photographie la radioactivité », sans perturber le milieu. Aucun radon ni descendant, ne sont prélevés dans la pièce, au cours de la mesure. Par ailleurs, les DSTN peuvent être conservés et relus plus tard. Enfin, ils ne ramènent au laboratoire, que l'image de la radioactivité et pas la radioactivité elle-même.

Tous les résultats (nb traces, diamètres, bruit de fond, etc.) d'un DSTN dépendent beaucoup des conditions de traitement et de comptage. En routine, le compromis adopté par chaque laboratoire est très important car il devra convenir à tous les DSTN traités. Aussi bien pour les faibles que pour les fortes expositions. Dans tous les cas, une densité de traces bien homogène est le signe d'une bonne maîtrise de la mesure et d'une moindre fluctuation des résultats.

Toutes les détections qui utilisent des DSTN, sont limitées en précision. En effet le nombre de traces observé $N_{\mathrm{T}}$ est une variable statistique. Au mieux, $95 \%$ des mesures se situent entre $N_{\mathrm{T}} \pm 2 \sqrt{ } N_{\mathrm{T}}$. Les erreurs expérimentales ne peuvent que réduire ce pourcentage théorique.

\section{Deux types de plastiques}

\section{I. DSTN « massifs »}

Historiquement de très nombreux DSTN « massifs » ont été utilisés. Les plus utilisés aujourd'hui sont des « polycarbonates » (CR39, Makrofol, etc.). Ils ressemblent 
à de petits morceaux de plexiglas, plus ou moins souples, de l'ordre du millimètre d'épaisseur. Les traces obtenues ressemblent à de petits cratères de surface, de différentes tailles.

Ils peuvent atteindre une grande sensibilité ( $\mathrm{nb}$ traces $/ \mathrm{cm}^{2}$ par $\mathrm{kBq} \mathrm{h} / \mathrm{m}^{3}$ ), qui dépend beaucoup du traitement. En règle générale, plus la sensibilité est grande, plus les traces sont larges et plus la saturation est faible. Par exemple, les traitements électrochimiques donnent plus de traces, mais qui sont plus larges.

\subsection{DSTN en couches minces}

Il s'agit uniquement du film Kodak LR115. Il est constitué d'une couche de nitrocellulose, fortement teinté en rouge, de $12 \mu \mathrm{m}$ d'épaisseur, sur un support inerte de $100 \mu \mathrm{m}$, en polyester. C'est le résultat de plus de 25 ans d'expérience de Kodak, au service de la détection des particules ionisantes.

Après traitement, chaque impact d'alpha laisse un vrai micro-trou dans la couche rouge, de 1 à $15 \mu \mathrm{m}$ de diamètre (contre $\approx 5$ à $80 \mu \mathrm{m}$ pour les DSTN massifs, selon leur mode de traitement). Au microscope, le film LR115 ressemble à une cible de tir! (Fig.1).

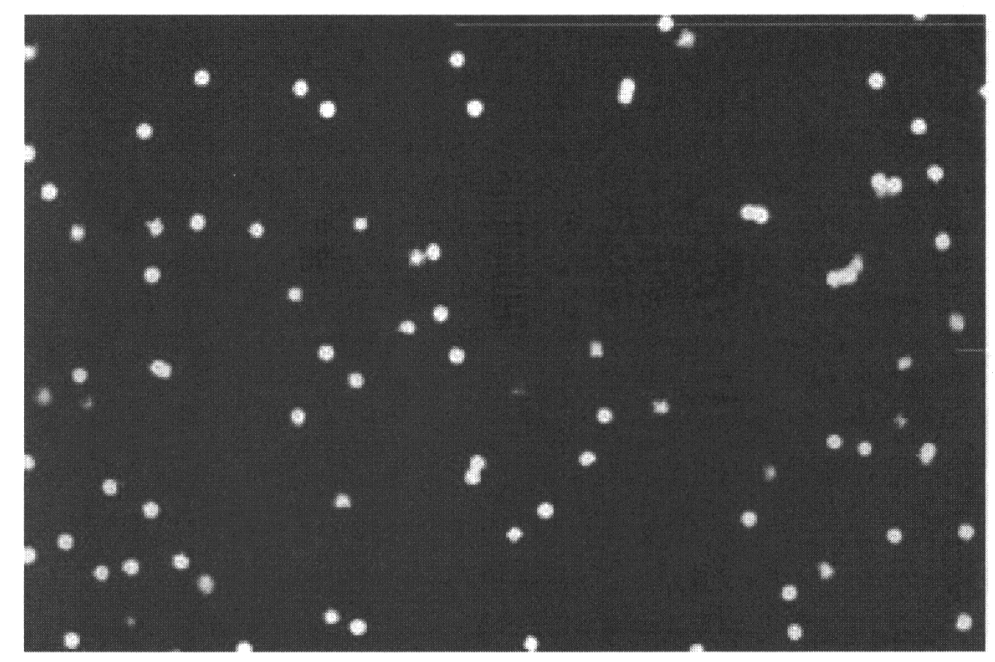

Fig. 1. - Traces de particules alpha sur un film Kodak LR115 (Fond rouge).

Alpha tracks on a Kodak LR115 film (red background). 
Il existe 2 types de LR115. Si la couche est «non pelliculable », les trous sont comptés optiquement. Si la couche est dite «pelliculable », elle est décollée de son support après traitement et on fait un comptage électrique des traces, par «claquages » entre 2 électrodes planes. L'avantage principal est de pouvoir utiliser de grandes surfaces de film, donc d'atteindre de grandes sensibilités. Par ailleurs, des appareils de claquage existent sur le marché. Par contre, la saturation est assez faible, de l'ordre de 10 à 40 traces $/ \mathrm{mm}^{2}$

\section{Différences par rapport aux DSTN massifs}

- Des traitements chimiques plus courts : $1 \mathrm{~h} 30$ à $2 \mathrm{~h}$, contre 3 à $8 \mathrm{~h}$.

- Aucune possibilité de confusion entre traces, poussières, rayures et défauts de surface. En effet, une trace est un trou, alors qu'une poussière absorbe la lumière. Ce qui permet l'emploi de logiciels de comptage optique, plus simples, plus sûrs et plus rapides.

- Possibilité de correction automatique des petits écarts inévitables de température $\left({ }^{\circ} \mathrm{C}\right)$ et de concentration du traitement, à partir de l'épaisseur résiduelle du film. Le logiciel de comptage peut même la déterminer automatiquement, par la mesure de la densité optique résiduelle du film. Les films traités doivent être vernis, avant comptage, pour éviter la diffusion de lumière produite par la rugosité de surface et par les trous eux-mêmes.

- Pour le LR115 non pelliculable, la saturation des comptages optiques est nettement plus élevée, de l'ordre de 600 traces $/ \mathrm{mm}^{2}$, contre $\approx 50$ à 400 traces $/ \mathrm{mm}^{2}$ pour les DSTN massifs, selon leur traitement. Une saturation trop basse devient très vite un handicap, en pratique.

- En plus d'une énergie minimum, le film LR115 possède une énergie alpha maximum, au-dessus de laquelle il n'y a pas formation de trace. Cette limite haute est typiquement de $4,5 \mathrm{MeV}$. Elle augmente un peu pour des traitements prolongés.

- Pour chaque émetteur, il existe une distance minimum, au dessous de laquelle aucune trace n'est possible : $5 \mathrm{~mm}$ pour le radon, $30 \mathrm{~mm}$ pour son descendant le plus énergétique. Ce qui fait que le LR115 est insensible aux poussières radioactives qui peuvent se déposer à sa surface.

- La protection contre l'augmentation du bruit de fond, est simple et très efficace. La simple auto-protection spire-spire des bobines, ou l'application d'un écran polyester épais contre le LR115, sont excellents.

Par contre, le film a un inconvénient pratique, qui peut parfois être gênant. Il est sensible à un excès de lumière solaire. Les UV accélèrent l'attaque de la soude et la dissolution totale de la couche est possible, en cas de trop forte exposition UV. Pour des mesures à l'air libre, surtout en extérieur, une protection efficace contre un excès de soleil est impérative. 


\section{Deux types de boîtiers détecteur}

\subsection{Les dosimètres de "type fermé »}

Un dosimètre de « type fermé » est un petit boîtier fermé, qui contient un DSTN. Seul le gaz radon peut entrer dans le boîtier, soit en passant par un filtre, soit en passant par de micro chicanes. Le nombre de traces dépend donc uniquement du radon qui est entré. À l'intérieur, tous les émetteurs atteignent rapidement l'équilibre radioactif. Comme il y a deux descendants, l' activité totale est 3 fois celle du radon. Mais un certain pourcentage des émetteurs présents, n'est pas enregistré, car les $\alpha$ émis sont parfois trop « obliques » par rapport au DSTN. Pour le radon cette proportion dépend surtout de la géométrie du boîtier, mais pour les descendants, elle est soumise aux caprices du statisme. En effet, tous les descendants se fixent « quelque part », sur les parois et sur le DSTN lui-même. C'est la principale cause des fluctuations du nombre de traces.

Le LR115, utilisé dans des boîtiers fermés, conserve ses avantages de DSTN en couche mince. Il est cependant préférable d'ajouter un écran « ralentisseur » contre le film, pour ralentir les émissions alpha des émetteurs trop proches du film. Cela permet même d'ajuster la sensibilité souhaitée. Cette contrainte est compensée par le fait que cet écran peut être antistatique, voire métallique et produire ainsi une meilleure homogénéité de la densité des traces. Un traitement antistatique est d'ailleurs conseille pour tous les DSTN.

Une parfaite étanchéité au radon est quasiment impossible. Même en production, ces dosimètres posent un problème de finition, puisqu'il faudrait fermer les emballages, dans des salles sans radon, ou sous vide. Quelques mois après leur production, le bruit de fond interdit déjà la mesure des faibles radioactivités (Mellander et Enflo, 1992). Les dosimètres « fermés » ont donc une date de péremption courte.

Ces dosimètres ne fonctionnent pas ON/OFF. À l'ouverture du boîtier, il faut environ 3 heures pour atteindre l'équilibre du radon dans et hors du boîtier ainsi que l'équilibre radioactif dans le boîtier. Mais surtout, en fin d'exposition, l'utilisateur est incapable de fermer efficacement le boîtier. Il faut donc procéder le plus vite possible à l'analyse, pour pouvoir négliger les expositions ultérieures. En routine, ces dosimètres mesurent donc difficilement les faibles expositions. Pour cette raison, des expositions inférieures à 2 mois ne sont pas conseillées.

Ces dosimètres posent aussi un problème d'accréditation. En effet, les essais d'accréditation, sont très éloignés des conditions de routine : dosimètres récents, délais courts d'emploi et de retour à l'analyse, ajout de dosimètres témoins, emballage de retour dans des plastiques soudés, etc. Sans une gestion quotidienne très contraignante de ces dosimètres, leur précision pratique risque d'être nettement moins bonne que pour les tests.

Malgré des principes simples, un bon DSTN dans un bon boîtier ne donne pas forcément un bon dosimètre. Le savoir faire du laboratoire fait toute la différence, par une multitude de petits détails pratiques que seule l'expérience apporte. 


\subsection{Les dosimètres de type "ouvert»}

Un dosimètre de «type ouvert » est un petit dispositif où le DSTN est exposé à l'air libre, dans le local à recontrôler. Seul le film LR115 convient à ces mesures, car il n'est pas sensible aux émetteurs collés à sa surface. Un dosimètre « ouvert » fonctionne ON/OFF. En position OFF, le LR115 est totalement protégé contre toute exposition, par un écran polyester.

En position ON, le film est à l'air libre, mais le volume de mesure est autolimité par les distances minimale et maximale auquel chaque émetteur est soumis. Les émetteurs de grande énergie sont mesurés dans un plus grand volume, mais globalement plus éloigné du film, donc avec une probabilité moindre d'atteindre le film (angle solide). En pratique, l'efficacité d'enregistrement de ces émetteurs est juste un peu plus élevée. Pour les autres DSTN, l'écart de sensibilité entre les divers émetteurs serait trop grand, puisqu'ils ne sont soumis qu'à une distance maximum, pour leur enregistrement.

Le nombre de traces obtenu est exactement proportionnel à la somme des activités des émetteurs alpha présents dans le volume de mesure. Il n'y a aucun problème de descendants déposés dans un coin, aucun problème de statisme du bốtier. Pour exprimer un résultat en terme de radon, il suffit de supposer que $F=0,40$. Ni plus ni moins que ce que supposent les recommandations elles-mêmes, pour exprimer en radon une recommandation initiale en énergie.

En pratique, comme les deux hypothèses des recommandations $(F=0,40$ et absence de thoron), sont souvent vérifiées, les bons dosimètres « ouvert » et « fermé » donnent exactement les mêmes résultats, dans $95 \%$ des cas. Les $5 \%$ restants, provoquent la tendance observée, de donner des résultats moyens un peu supérieurs aux dosimètre "fermés ». Mais, si les calibrations sont bonnes et si la moyenne des facteurs d'équilibre rencontrés est bien de 0,40 , cela révèle une présence de thoron dans certains locaux. Devrait-on laisser les gens dans leur thoron, uniquement parce que les recommandations n'en parlent pas encore?

Lorsqu'un dosimètre « ouvert » donne un résultat significativement supérieur à un dosimètre « fermé », c'est que le risque est vraiment supérieur, à cause d'un $F$ élevé ou d'une présence de thoron. D'ailleurs, les recommandations elles-mêmes, en $\mathrm{Bq} / \mathrm{m}^{3}$, ne sont équivalentes aux recommandations en Joules $/ \mathrm{m}^{3}$, donc à un risque, que pour $F=0,40$.

Le seul problème technique est de s'assurer que la sensibilité à $F$ et au thoron, n'est pas exagérée, par rapport aux recommandations en Joules.

La figure 2 montre l'écart entre la stricte concentration de radon et le résultat fourni par un dosimètre fermé, quand $F$ vrai est différent de 0,40 . Le premier étalonnage expérimental date d'une étude de 1991 de l'US-EPA (Yeager et al., 1991). 


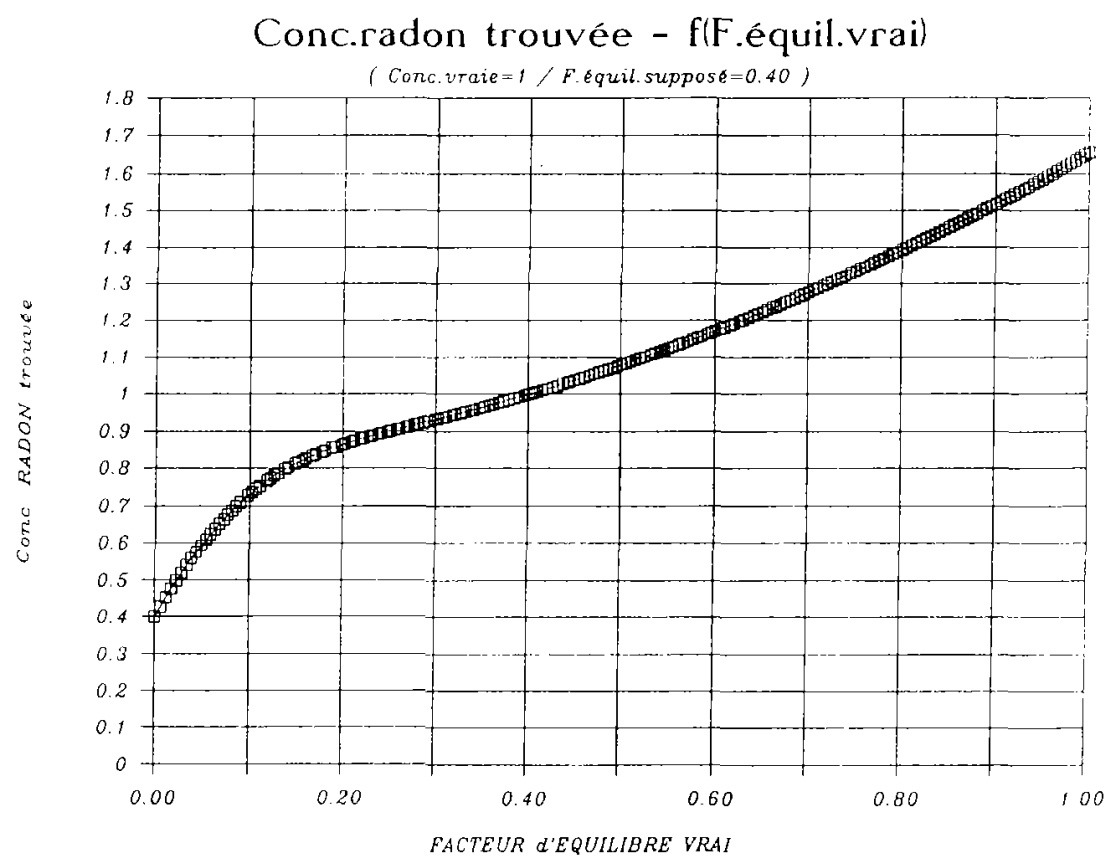

Fig. 2. - Concentration de radon trouvée par un dosimètre de type « ouvert ».

Radon concentration found by an "open type" detector.

Dans son rapport, elle concluait que les dosimètres « ouvert » permettaient même de déterminer le $F$ vrai, par simple comparaison entre les résultats de deux dosimètres « ouvert » et « fermé ». Ce qui a été fait de nombreuses fois depuis.

\subsection{Sensibilité au thoron des dosimètres de type «ouvert »}

Les diverses caractéristiques radioactives des radioéléments étant immuables, il est aisé et fiable de modéliser l'évolution dans le temps de l'activité des gaz et des descendants, des diverses EPAC et même du nombre de traces obtenues, en fonction de l'aération des bâtiments et des entrées des gaz radon et thoron.

En passant, on peut vérifier que les gaz radon et thoron ne sont pas des polluants ordinaires. Les pertes par décroissance radioactive limitent l'augmentation des concentrations, même à entrée constante du polluant. L'aération diminue ces concentrations d'équilibre mais accélère beaucoup leur atteinte. On peut s'apercevoir que le thoron n'est quasiment pas éliminable par aération (préférer aération 


\section{TABLEAU II}

Simulation : sensibilité au thoron, des dosimètres de types « ouvert » et « fermé ». (Air homogénéisé par ventilation. Même risque sanitaire radon et thoron)

Simulation: sensitivity to thoron of "open and close types" detectors. (Enough air mixing. Same health risk : radon and thoron).

\begin{tabular}{|c|c|c|c|}
\hline $\begin{array}{l}\text { AÉRATION } \\
\text { VARIABLE }\end{array}$ & $\begin{array}{l}\text { PARAMTitres } \\
\text { (a l'equillbre) }\end{array}$ & $\begin{array}{l}\mathrm{EPA}_{\mathrm{C}}=1 \mu \mathrm{Jm}^{3} \\
\mathrm{RADON}, 2 \mathrm{Rn}_{\mathrm{n}}\end{array}$ & 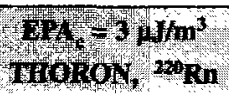 \\
\hline \multirow{5}{*}{ Aération $=0 \% / \mathrm{h}$} & $F /$ Temps d'équilibre $=$ & $1,00 / 2$ jours & $1.00 / 70 \mathrm{~h}$ \\
\hline & $C_{\text {vrai, gaz }} /$ Temps d'équilibre $=$ & $180 \mathrm{~Bq} \mathrm{~m}-3 / 25$ jours & $40 \mathrm{~Bq} \mathrm{~m} \mathrm{~m}^{-3} / 6 \mathrm{~min}$ \\
\hline & Entrée Constante $(\mathrm{gaz})=$ & $1,4 \mathrm{~Bq} \mathrm{~m}^{-3} \mathrm{~h}^{-1}$ & $1800 \mathrm{~Bq} \mathrm{~m}^{-3} \mathrm{~h}^{-1}$ \\
\hline & $C_{R n}$ mesuré 《 ouvert $»(F=0,4)=$ & $295 \mathrm{~Bq} \mathrm{~m}^{-3}$ & $75 \mathrm{~Bq} \mathrm{~m}^{-3}$ \\
\hline & $C_{\mathrm{Rn}}$ mesuré « fermé » $=$ & $180 \mathrm{~Bq} \mathrm{~m}^{-3}$ & $\cong 0 \mathrm{~Bq} \mathrm{~m}^{-3}$, variable \\
\hline \multirow[t]{5}{*}{ Aération $=10$ of th } & f Torips d'equilibre $=$ & $0.92 f 15 \mathrm{~h}$ & $0.39120 \mathrm{~h}$ \\
\hline & $C_{\text {nat, }}$ / Thaps d'Equilibre $=$ & $195 \mathrm{~Bq} \mathrm{~m}^{-3} / 45 \mathrm{~h}$ & $100 \mathrm{Bg} \mathrm{m}^{3} / 6 \mathrm{~min}$ \\
\hline & Entre Constante $(\mathrm{gaz})=$ & $21 \mathrm{~Bq} \mathrm{~m}^{3} \mathrm{H}$ & $4600 \mathrm{Bg} \mathrm{m}-1 \mathrm{~h}$ \\
\hline & nesure \& ouvert $\times(F=0.4)=$ & $300 \mathrm{~Bq} \mathrm{~m}^{3}$ & $140 \mathrm{Bg} \mathrm{n}^{3}$ \\
\hline & $C_{\text {R }}$ nesure \& fenme $=$ & $195 \mathrm{~Bq} \mathrm{\textrm {m } ^ { 3 }}$ & $=0 \mathrm{~Bq} \mathrm{~m}^{-3}$, vantable \\
\hline \multirow{5}{*}{$\begin{array}{l}\text { Aération }=100 \% / \mathrm{h} \\
\text { ( } \text { c classique } \% \text { ) }\end{array}$} & $F /$ Temps d'équilibre $=$ & $0,53 / 5 \mathrm{~h}$ & $0,06 / 4 \mathrm{~h}$ \\
\hline & $C_{\text {vrai, gaz }} /$ Temps d'équilibre $=$ & $340 \mathrm{~Bq} \mathrm{~m}^{-3} / 5 \mathrm{~h}$ & $700 \mathrm{~Bq} \mathrm{~m}{ }^{-3} / 6 \min$ \\
\hline & Entrée Constante $(\mathrm{gaz})=$ & $345 \mathrm{~Bq} \mathrm{~m}^{-3} \mathrm{~h}^{-1}$ & $32300 \mathrm{Bqm}^{-3} \mathrm{~h}^{-1}$ \\
\hline & mesuré « ouvert" $(F=0.4)=$ & $380 \mathrm{~Bq} \mathrm{~m}^{-3}$ & $770 \mathrm{~Bq} \mathrm{~m} \mathrm{~m}^{-3}$ \\
\hline & CRn mesuré « fermé »= & $340 \mathrm{~Bq} \mathrm{~m}^{-3}$ & $\cong 0 \mathrm{~Bq} \mathrm{~m}^{-3}$, variable \\
\hline \multirow{5}{*}{ 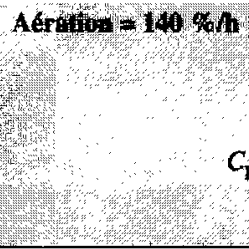 } & F Temps d'equilibre = & $0,4513 \mathrm{~h}$ & $0,043 \mathrm{~h}$ \\
\hline & $C_{\text {vad } g a x}$ / Temps dequitiore & $400 \mathrm{~Bq} \mathrm{~m}^{-3} / 3 \mathrm{~h}$ & $950 \mathrm{Bg} \mathrm{m}^{-3} / 6 \mathrm{~min}$ \\
\hline & Entree Constante (gaz) $=$ & $565 \mathrm{Bg} \mathrm{h} / \mathrm{h}$ & $44300 \mathrm{Eg} \mathrm{m}^{-3} \mathrm{~b}^{-1}$ \\
\hline & mesure \& ouver,$(\mathbf{P}=0,4)=$ & $415 \mathrm{~Bq} \mathrm{~m}^{-3}$ & $1060 \mathrm{~Bq} \mathrm{~m}^{-3}$ \\
\hline & $C_{\mathrm{R}}$ mesué $\alpha$ ferme $\#=$ & $400 \mathrm{~Bq} \mathrm{~m}^{3}$ & $=0 \mathrm{Bqm}^{-3}$, varubet \\
\hline
\end{tabular}

à ventilation). Mais comme cette aération élimine mieux ses descendants, le facteur d'équilibre $F_{\mathrm{T}}$ observé est presque toujours très faible $(\approx 0,05)$. Inversement, l'aération a un gros effet sur le risque radon. Elle réduit beaucoup la concentration du gaz, mais elle abaisse aussi $F_{\mathrm{R}}$. Deux causes de baisse de l'EPA $\mathrm{R}_{\mathrm{R}}$, donc du risque. Une aération classique de $100 \%$ par heure, abaisse déjà $F$ vers 0,50 . Les pertes par dépositions des descendants sur les cloisons, ne peuvent que s'ajouter à cette baisse de $F$.

Le tableau II est extrêmement riche en informations. Il compare, en fonction de l'aération, les entrées de gaz nécessaires pour atteindre une EPA ${ }_{\text {radon }}$ de $1 \mu \mathrm{J} / \mathrm{m}^{3}$ et une $\mathrm{EPA}_{\text {thoron }}$ de $3 \mu \mathrm{J} / \mathrm{m}^{3}$. Il est généralement admis que ces deux énergies correspondent à un même risque sanitaire. Toutes les valeurs fournies sont celles atteintes à l'équi- 
libre. Les temps d'atteinte sont aussi mentionnés. Les dépôts de descendants sur les parois sont négligés, dans un premier temps. L'air ambiant est supposé bien brassé.

Pour ce qui concerne la mesure du risque thoron, on constate que, pour une aération de $10 \% / \mathrm{h}$ la sensibilité au risque thoron des dosimètres « ouvert », est deux fois plus faible qu'au risque radon. En effet, on mesure $300 \mathrm{~Bq} / \mathrm{m}^{3}$ pour $1 \mu \mathrm{J} / \mathrm{m}^{3}$ de radon contre $140 \mathrm{~Bq} / \mathrm{m}^{3}$ pour $3 \mu \mathrm{J} / \mathrm{m}^{3}$ de thoron. On constate aussi que pour $100 \% / \mathrm{h}$, c'est l'inverse, la mesure surestime le risque d'un facteur 2 . La prise en compte des dépôts de descendants sur les parois, agraverait un peu cette tendance, mais un autre phénomène le réduirait beaucoup : l'absence de gaz thoron au centre des pièces peu ventilées (brassées), d'où augmentation du $F$ au centre et possibilité de peu de traces pour beaucoup de descendants.

Sur ce tableau, on retrouve, bien sûr, d'autres données plus classiques :

- Le produit constant $F \times C$ (gaz), puisqu'on travaille à EPA ${ }_{c}$ constante.

- Un gros effet sanitaire de l'aération sur le risque radon. Pour une aération classique de $100 \% / \mathrm{h}$, les entrées radon à $10 \% / \mathrm{h}$ doivent être multipliées par 16 , pour atteindre la même $\mathrm{EPA}_{\mathrm{c}}$. Par ailleurs $F$ tombe déjà à 0,53 , sans compter les dépôts sur les parois.

- L'élimination casi-nulle du thoron par aération, à cause de sa durée de vie de $50 \mathrm{~s}$.

- L'aération fait, par contre très vite baisser $F_{\text {thoron, }}$ donc le risque. Il faut alors d'énormes entrées pour atteindre nos $3 \mu \mathrm{J} / \mathrm{m}^{3}$.

$-e t c$.

Dans une pièce où l'air serait brassé, le risque thoron serait donc surestimé par deux. Cependant, en pratique, le gaz thoton est nettement plus concentré près de sa source et la sensibilité au risque thoron peut être sous-estimée jusqu'à 5 fois, par des mesures « ouvertes » éloignées des murs.

\subsection{Passage progressif du dosimètre " ouvert » au dosimètre «fermé \#}

Le tableau III montre qu'on peut imaginer des dosimètres intermédiaires, qui peuvent même parfois cumuler les avantages des uns et des autres.

On peut envisager d'autres configurations de dosimétrie fermés, basées sur le LR115, qui conservent les avantages de la détection ouverte (en particulier ON/OFF) tout en étant de type « fermée ».

Dans un « gobelet calibré», le facteur d'équilibre est d'environ 0,05. L'entrée de gaz se fait uniquement par la non-étanchéité du couvercle. Quand on perce de plus en plus de gros trous dans le gobelet, le $F$ à l'intérieur devient soudainement égal au $F$ à l'extérieur. Le nombre de traces obtenues devient alors égal à ce qu'on obtient avec un dosimètre ouvert. 
TABLEAU III

Passage des dosimètres de type « ouvert (A) aux dosimètres de type « fermés » (D). Dosimeters, from "open type" (A) to "close type" (D).

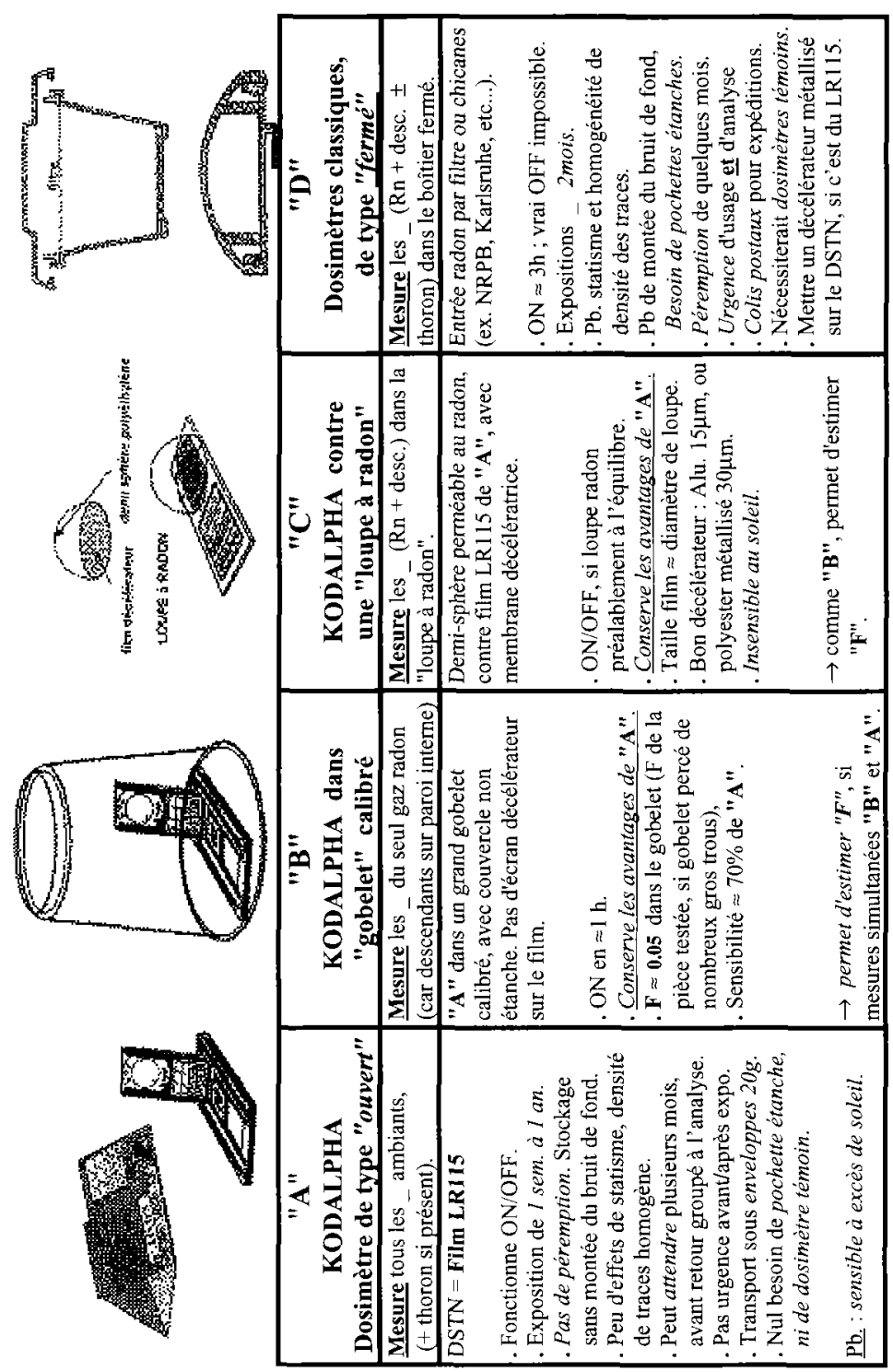


Faire de la détection «fermée » avec des films qui seront traités en même temps que ceux d'une détection « ouverte », permet une bien meilleure estimation de $F$. En effet, les petits écarts de traitements, donc de sensibilité, sont alors les mêmes et ne perturbent pas leur rapport. DOSIRAD utilise déjà les « gobelets calibrés » dans lesquels on place des dosimètres " ouvert ». DOSIRAD travaille aussi à la mise au point de la « loupe à radon », une petite « chambre » externe au film LR115, qui transforme un dosimètre ouvert en un dosimètre fermé.

Mais, nous souhaitons continuer à promouvoir la détection « ouverte »; qui demeure la meilleure façon d'estimer le risque global radon, au meilleur prix, lors d'une première mesure.

\section{Références}

Brochure (1996) Le radon de l'O.M.S.

Cliff K.D., Green B.M.R., Mawle A., Miles J.C.H. (1992). Radiation Protection Dosimetry, 45, 361-366.

Collectif de l'IPSN (1996), Le radon 222 et ses descendants à vie courte dans ]'environnement atmosphérique : origine et méthodes de mesures. Radioprotection, 31, 371 - 388.

ICRP (1994) International Commission on Radiological Protection, tableau 5, p.17, tableau 7 et 8 p. 23.

Journal officiel des Communautés Européennes (1990) 90/143/Euratom.

Li Yanxia, Schery S.D. (1992) Measurements of Indoor Thoron Levels and Disequilibrium Factors. New Mexico Institute of Mining and Technology, Sorocco, Minneapolis, Minnesota, USA, International Symposium on Radon.

Mellander H., Enflo A. (1992) The alpha track method used in the Swedish radon epidemiological study. Radiation Protection Dosimetry, 4, $65-71$.

Steinhäusler F. (1995) Gas Geochemistry, The significance of the exposure to thoron decay products., University of Salzburg, Institute of Physics and Biophysics, Austria.

Tymen G., Mouden A., McLaughlin J.P., Wasiolek P., Rannou A. (1988) A comparison of the exposure response of French and Irish radon detectors during field measurements in houses in Britanny. Radiation Protection Dosimeters, 24, 371 - 374.

Yeager W., Boyd M.A., Popell S.W. Jr. (June 1991) A performance évaluation of unfiltered alpha track detectors. US-EPA contract 68D90064. 